\title{
Automatic Generation of Virtual Computer Rooms on the Internet Using X3D
}

\author{
Aybars Uğur ${ }^{1}$ and Tahir Emre Kalayci ${ }^{2}$ \\ ${ }^{1}$ Ege University, 35100 Bornova-Izmir, Turkey, \\ aybars .ugur@ege.edu.tr \\ 2 tahir.kalayci@ege.edu.tr \\ http://yzgrafik.ege.edu.tr
}

\begin{abstract}
In this paper, some natural links between virtual reality and interactive 3D computer graphics are specified and Web3D technologies especially VRML and X3D are briefly introduced. Web-based tool which is called EasyLab3D was designed and implemented using X3D. This web-based tool is used to generate automatically $3 \mathrm{D}$ virtual computer rooms that can be navigated in. It is important for introduction of departments, companies and organizations which have computer laboratories and for planning new computer rooms. As a result, state-of-the art technologies and methods in development of automatic 3D scene and model generation tools are discussed.
\end{abstract}

\section{Introduction}

Sherman and Craig[1] define VR (Virtual Reality) as "a medium composed of interactive computer simulations that sense the participant's position and actions and replace or augment the feedback to one or more senses, giving the feeling of being mentally immersed or present in the simulation". According to them, the key elements to experiencing VR are a virtual world, immersion, sensory feedback, and interactivity. It is the use of computer graphics systems in combination with various display and interface devices to provide the effect of immersion in the interactive 3D computer-generated environment. We call such an environment a virtual environment 2].

High quality interactive $3 \mathrm{D}$ content on the web without bandwidth and platform limitations allows internet users to become fully immersed in realistic 3D worlds. Many Web3D technologies have been developed to give people real-time, three-dimensional and interactive computer graphics on the web.

In this study, we designed and implemented an X3D-based tool (EasyLab3D) which is used to generate automatically and navigate in virtual computer rooms on the web using state-of-the-art technologies.

\section{Extensible 3D (X3D)}

Web3D is simply 3D graphics on the web. VRML-NG (X3D) arises in 1999 as a result of efforts to carry 3D to all environments. X3D which is developed 
by Web3D consortium is the ISO standard for real-time 3D computer graphics. XML was adopted as syntax for X3D in order to solve a number of real problems of VRML. According to Yumetech President, Alan Hudson the reasons to use XML as syntax are to interoperate with the Web and to incorporate new graphics technologies in a standardized way 1 . Main X3D features are extensions to VRML (e.g. Humanoid Animation, Nurbs, GeoVRML etc.), the ability to encode the scene using an XML syntax as well as the Open Inventor-like syntax of VRML97, and enhanced application programmer interfaces.

Blais et al. 3] present a Web3D application for military education and training. Patel et al. 4] describe an innovative system designed for museums to create, manage and present multimedia based representations of museum artifacts in virtual exhibitions both inside and outside museums. Barbieri et al. [5] developed computer science virtual museum which can be visited online, also which contains simple interactive games that illustrate basic principles of computer science. Some other projects based on X3D are explained in [6] and [7].

\section{Automatic Computer Room Generation Tool}

We developed Web-based Automatic Virtual Computer Room Generation Tool (EasyLab3D2) using Java and Xj3D. Some features such as navigation in 3D room scenes and realistic presentation are important for people to introduce computer laboratories to visitors or internet users. This tool can also be used for designing new labs by providing 3D previews. 3D model of a computer laboratory (Ege Lab) is generated using EasyLab3D in a few seconds (Fig. 1).

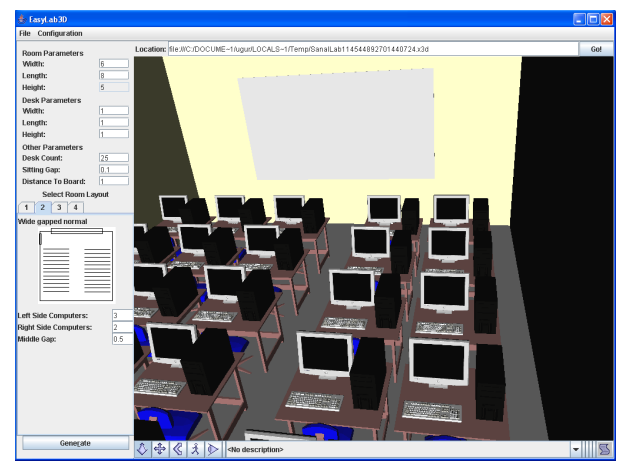

Fig. 1. Ege Lab in our department generated by EasyLab3D (Perspective View)

3D models (table/desk, room) required by program were developed using Flux Studio 2.0 Beta 3 . All models were developed as prototype to reduce the file size

\footnotetext{
${ }^{1}$ http://www.xml.com/pub/a/2003/08/06/x3d.html

${ }^{2}$ http://yzgrafik.ege.edu.tr/projects/easylab3d/

${ }^{3}$ http://www.mediamachines.com/make.php
} 
and to use an object many times without coding same things repeatedly. Created model prototypes are stored at web spact 4 for online use.

Scenes are generated using our layout algorithms and prototype instances are created with calculated transformations and put in a temp file on the fly. Thus user can save the scene generated as a file and publish on the web site. User can also examine these files later using other browsers and plug-ins. Scenes are created on the fly, shown on the Xj3D browser using Java programming language. Xj3D provides to change X3D scenes dynamically using SAl5 (Scene Authoring Interface) technology. Java Web Start technology 6 is used to make easier run of program and to be downloaded all required libraries automatically.

Automatic generation algorithms for most popular rectangular computer room layouts are implemented in this project. Computers which are on the tables are placed in that order. Computer Room Layout 1 has equally sized gaps between computer tables and Layout 2 includes one corridor has two computer blocks - one at the left side of room and one at the right side shown in Fig. 2 , Room parameters given by the user are width (sizeX), length (sizeY), height and distance to board (gapY, minimum feasible distance between board and tables). Table/desk parameters are width (sizeX, width of table), length (sizeY) and height. Some other parameters are Desk Count (room.tableCount), Sitting Gap (table.gapY, feasible distance between tables that a computer user works easily, in $\mathrm{Y}$ axis), Desk Gap (table.gapX, gap between tables in X axis).

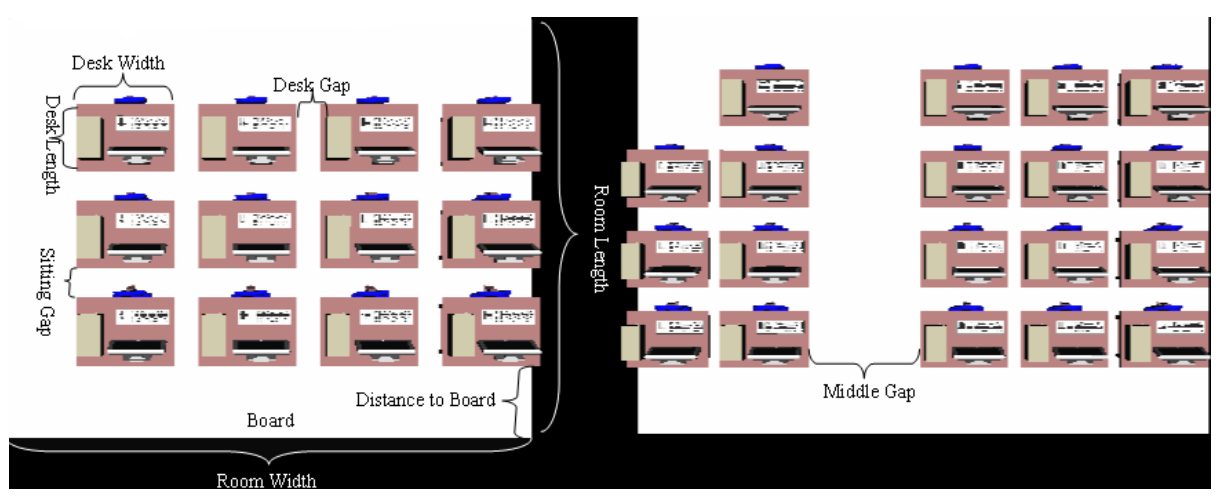

Fig. 2. Computer Room Layout 1 (on the left) and Layout 2 (on the right)

Java-like Algorithm generates 3D model of computer room for Layout 1:

// Calculating table capacity of room width (X) room.tCountX = (room.sizeX+table.gapX)/(table.sizeX+table.gapX);

// Calculating table capacity of room length (Y) room.usableLength = room.sizeY - room.gapY;

\footnotetext{
${ }^{4}$ http://yzgrafik.ege.edu.tr/elab/modeller/

${ }^{5}$ http://www.xj3d.org/tutorials/general_sai.html

${ }^{6}$ http://java.sun.com/products/javawebstart/
} 


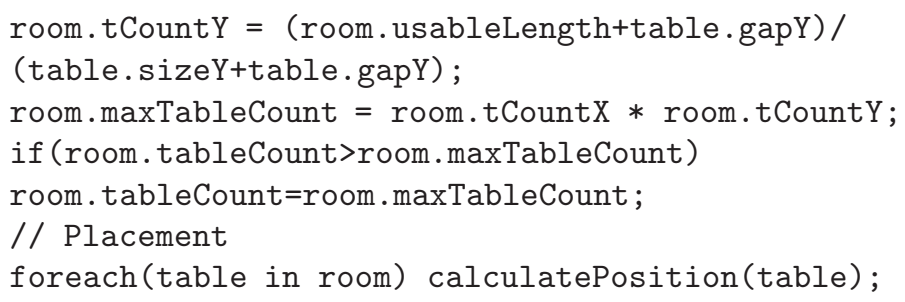

Tables and computers exceeding the room capacity are not inserted into the scene as specified in the algorithm.

\section{Conclusion}

New generation 3D languages, APIs and key Web3D technologies (X3D, Java 3D) offer possibilities for creating and manipulating with 3D objects and scenes easily. X3D provides support to insert 3D shapes, text and objects into scene, to transform objects, to modify attributes of objects and others such as grouping, animation, illumination, etc. X3D also uses XML's advantages such as rehostability, page integration, integration with the next-generation web technologies, extensive tool chain support. Developments in Web3D technologies and authoring tools are important for the future of VR.

Increasing number of web-based 3D graphics projects easy to use like EasyLab3D will make 3D graphics natural part of web and will improve web quality. $3 \mathrm{D}$ interactive model of a computer lab is more than a series of $2 \mathrm{D}$ pictures and also more enjoyable and realistic. Companies and organizations can use this tool for generating 3D models of their labs only giving a few parameters. Internet users can access and navigate in these lab models. This automatic generation tool is also useful for interior designs of new labs by providing 3D previews.

\section{References}

1. Sherman, W.R., Craig, A.B.: Understanding Virtual Reality: Interface, Application, and Design. Morgan Kaufmann Publishers Inc., San Francisco, CA, USA (2002)

2. Sowizral, H.A., Deering, M.F.: The java 3d api and virtual reality. IEEE Computer Graphics and Applications 19 (1999) 12-15

3. Blais, C., Brutzman, D., Horner, D., Nicklaus, S.: Web-based 3d technology for scenario authoring and visualization: The savage project. In: I/ITSEC. (2001)

4. Patel, M., White, M., Walczak, K., Sayd, P.: Digitisation to presentation - building virtual museum exhibitions. In: VVG. (2003) 189-196

5. Barbieri, T., Garzotto, F., Beltrame, G., Ceresoli, L., Gritti, M., Misani, D.: From dust to stardust: A collaborative 3 d virtual museum of computer science. In: ICHIM (2). (2001) 341-345

6. Hetherington, R., Farrimond, B., Presland, S.: Information rich temporal virtual models using x3d. Computers \& Graphics 30 (2006) 287-298

7. Yan, W.: Integrating web $2 \mathrm{~d}$ and $3 \mathrm{~d}$ technologies for architectural visualization: applications of svg and x3d/vrml in environmental behavior simulation. In: Web3D '06: Proceedings of the eleventh international conference on 3D web technology, New York, NY, USA, ACM Press (2006) 37-45 